\title{
Value of a quantitative model of axillary venous blood flow spectrum for the detection of central venous stenosis in patients undergoing hemodialysis via radiocephalic arteriovenous fistula
}

\author{
Xiang $\mathrm{Xu}^{1}{ }^{\wedge}$, Yong Zhuang ${ }^{1}$, Jinshu Zeng ${ }^{1}$, Fanggang $\mathrm{Cai}^{2}$, Tianmin $\mathrm{He}^{2}$, Jie $\mathrm{Wu}^{2}$, Caiming Chen ${ }^{3}$, \\ Zhenhuan Zou ${ }^{3}$, Xiaohong Zhang ${ }^{3}$, Guorong $\mathbf{L v}^{4,5}$ \\ ${ }^{1}$ Department of Ultrasound, the First Affiliated Hospital of Fujian Medical University, Fuzhou, China; ${ }^{2}$ Department of Vascular Surgery, the \\ First Affiliated Hospital of Fujian Medical University, Fuzhou, China; ${ }^{3}$ Department of Nephrology, the First Affiliated Hospital of Fujian \\ Medical University, Fuzhou, China; ${ }^{4}$ Department of Ultrasound, the Second Affiliated Hospital of Fujian Medical University, Quanzhou, China; \\ ${ }^{5}$ Collaborative Innovation Center for Maternal and Infant Health Service Application Technology, Quanzhou Medical College, Quanzhou, China \\ Contributions: (I) Conception and design: G Lv, X Xu; (II) Administrative support: G Lv; (III) Provision of study materials or patients: F Cai, T He, \\ J Wu, C Chen, Z Zou, X Zhang; (IV) Collection and assembly of data: Y Zhuang, J Zeng; (V) Data analysis and interpretation: X Xu, Y Zhuang, J \\ Zeng; (VI) Manuscript writing: All authors; (VII) Final approval of manuscript: All authors. \\ Correspondence to: Guorong Lv. Department of Ultrasound, the Second Affiliated Hospital of Fujian Medical University, 34 Zhongshan North \\ Road, Quanzhou 362000, China. Email: lgr_feus@sina.com; Xiang Xu. Department of Ultrasound, the First Affiliated Hospital of Fujian Medical \\ University, 20 Chanzhong Road, Fuzhou 350005, China. Email: riverpicture@163.com.
}

Background: Central venous stenosis (CVS) of radiocephalic arteriovenous fistula (RCAVF) affects RCAVF function and longevity. Ultrasound screening for CVS is limited by acoustic window. Herein, we analyzed the quantitative axillary venous $(\mathrm{AxV})$ spectrum in hemodialysis patients via RCAVF, and constructed central venous stenosis index (CVSI) model based on the spectrum parameters to early detect resting asymptomatic CVS.

Methods: From August 2017 to May 2021, stage 5 chronic kidney disease (CKD) patients dialysed via RCAVF at the First Affiliated Hospital of Fujian Medical University were included in this study. No CVSrelated symptoms were found and the pulsation at the arteriovenous anastomosis was normal. However, the patients had the sensation of swelling in the ipsilateral upper limb during dialysis; the venous pressure advanced upon the completion of dialysis; or both $(n=52)$. The inclusion criteria were as follows: (I) Ultrasound (US) showed that the temporal phases of the AxV spectrum were "normal"; and (II) CVS was confirmed by digital subtraction angiography (DSA). The exclusion criteria were as follows: (I) stent placement; (II) multiple stenosis; and (III) placement of central venous catheter. A total of 37 patients participated in the analysis. Eighteen patients were included in the CVS group, and 19 cases without CVS were included in the control group. Independent sample $t$-test was used to screen each parameter of the $\mathrm{AxV}$ spectrum, and a CVSI model was constructed by principal component analysis (PCA). The receiver operating characteristic curve (ROC) was applied to analyze the diagnostic value of CVSI.

Results: According to the independent sample $t$-test, 9 parameters were found to have statistical significance (all $\mathrm{P}<0.05$ ); they were analyzed by PCA, and the CVSI model was constructed. The ROC showed that CVSI had diagnostic value for CVS. When the cut-off value of CVSI was 7.13, the maximum value of the Youden index was 0.842 , with a sensitivity of $100 \%$ and a specificity of $84.2 \%$.

Conclusions: The CVSI helps to early detect resting asymptomatic CVS and dramatically increases the detection rate of CVS.

Keywords: Central veins; stenosis; ultrasound; model; arteriovenous fistula

\footnotetext{
$\wedge$ ORCID: 0000-0002-2636-7586.
} 
Submitted Sep 28, 2021. Accepted for publication Jan 13, 2022.

doi: $10.21037 / \mathrm{atm}-22-160$

View this article at: https://dx.doi.org/10.21037/atm-22-160

\section{Introduction}

End-stage renal disease (ESRD) caused by chronic kidney disease (CKD) has a poor prognosis, and hemodialysis is currently the main treatment modality. Both the National Kidney Foundation/Disease Outcomes Quality Initiative (NKF/DOQI) and the European Society for Vascular Surgery (ESVS) clinical practice guidelines for vascular access recommend arteriovenous fistula (AVF) as the preferred vascular access for hemodialysis $(1,2)$. The patency vascular access (including the feeding arteries, arteriovenous anastomoses, draining peripheral veins, and central veins) becomes the lifeline in ESRD patients, and the central veins (including the subclavian vein, innominate vein, and superior vena cava) are the last critical links in this lifeline.

The incidence of central venous stenosis (CVS) is approximately $10 \%$ in the general population, and up to $43 \%$ of CVS patients have a history of hemodialysis (3). The presence of CVS can seriously affect the function and longevity of the affected vascular access, and symptomatic CVS can lead to increased hospitalization and mortality in hemodialysis patients. Therefore, early detection of CVS facilitates the development of a treatment strategy for CVS, which is essential for preserving the function of AVF (4).

The diagnosis of CVS mainly relies on clinical manifestations and imaging findings. Clinically, CVS manifests as elevated central venous pressure (edema of the arms, head, face, and chest as well as dilated or varicose veins in the chest wall); however, some CVS patients can be asymptomatic and physically normal at rest, even in the presence of increased venous pressure in the vascular access during hemodialysis, especially in those using AVF as the vascular access (5). In addition, the increased venous pressure during dialysis does not provide localization and diagnosis of problems in the central veins, and CVS is often neglected due to the lack of specificity $(6,7)$. The gold standard for assessing CVS is digital subtraction angiography (DSA), which, however, cannot be used for routine vascular access monitoring and examination due to its invasiveness. In contrast, color Doppler ultrasonography (CDU) has been used for the initial imaging of hemodialysis access and is applied for hemodynamic monitoring (8). When CDU diagnoses CVS, it is mainly via detection of the changes in the lumen and flow velocity at the CVS site (9); however, the central veins are located posterior to the sternum and clavicles and the acoustic shadows of bones and lungs can interfere with the display of superior vena cava, innominate vein, and subclavian vein on CDU (10). Although Sivanesan et al. (11) used quantitative model to study AVF, their study was a basic study based on AVF model in vitro. In this study, a quantitative model of anastomotic hemodynamics was established to explore the pathogenic role of anastomotic geometry and local hemodynamics changes in AVF stenosis. This study is not suitable for the detection of asymptomatic central venous stenosis. In addition, although computed tomography angiography (CTA) and medical resonance angiography (MRA) have better diagnostic performance than that of CDU, they are not as popular as CDU and DSA because the contrast agents used can cause damage to residual normal renal tissue in ESRD patients. In addition, unlike DSA, these techniques do not enable immediate subsequent interventions after the detection of stenosis $(12,13)$. In this study, we retrospectively analyzed the quantitative data of axillary venous flow spectrum in stage 5 CKD patients undergoing hemodialysis via a forearm radiocephalic arteriovenous fistula (RCAVF) and found that the axillary venous spectrum changed when CVS occurred. A central venous stenosis index (CVSI) evaluation model was constructed based on the quantitative axillary venous spectrum parameters to facilitate noninvasive early detection of resting asymptomatic CVS.

We present the following article in accordance with the STARD reporting checklist (available at https://atm. amegroups.com/article/view/10.21037/atm-22-160/rc).

\section{Methods}

\section{Participants}

From August 2017 to May 2021, stage 5 CKD patients receiving regular hemodialysis via RCAVF at the First Affiliated Hospital of Fujian Medical University were included in this study. This study adhered to the tenets of the Declaration of Helsinki (as revised in 2013). This study was approved by the Ethics Committee of the First Affiliated Hospital of Fujian Medical University [MRCTA, ECFAH of FMU (2020)189]. Informed consent to review 


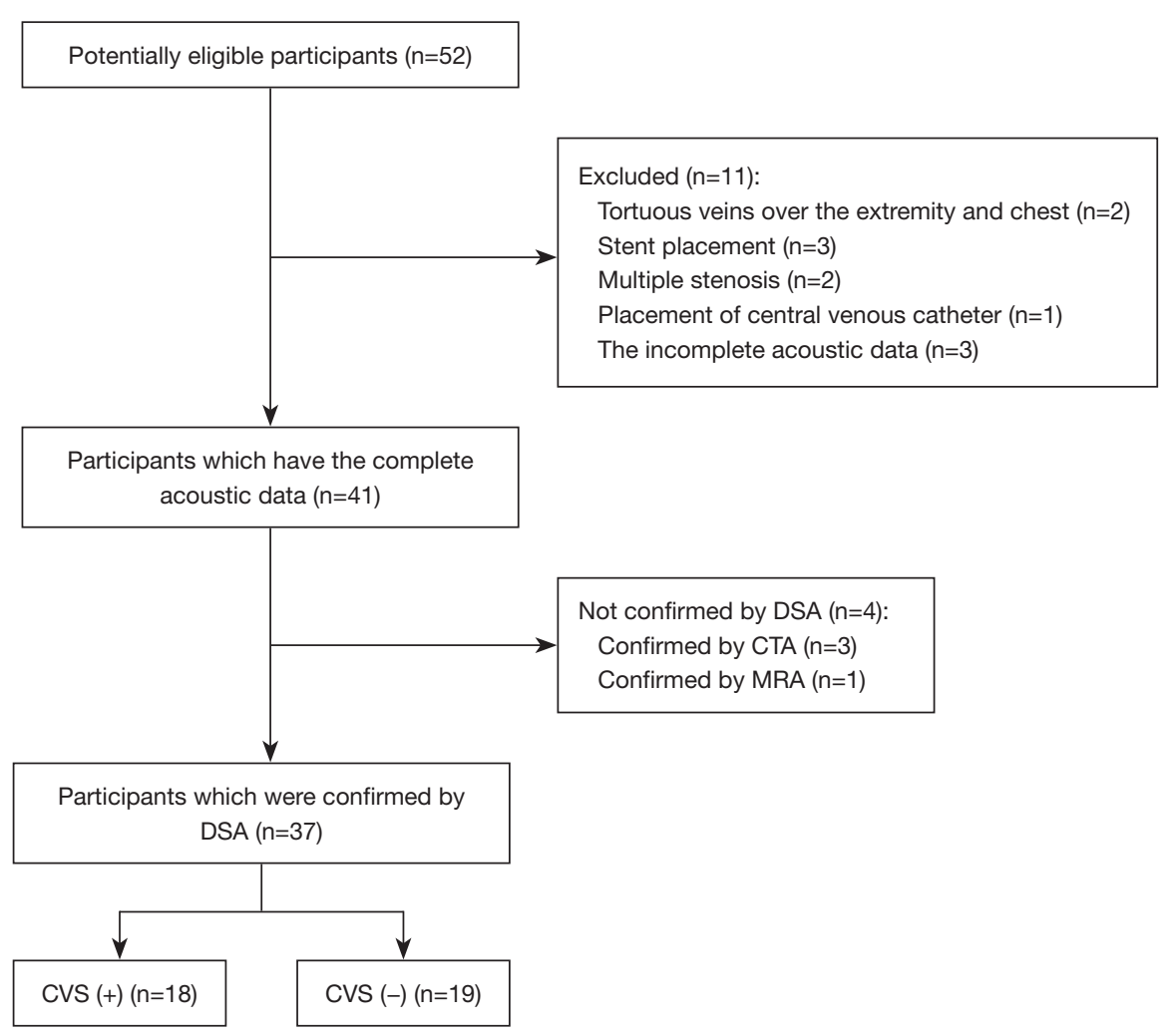

Figure 1 Flow chart of participant enrollment. DSA, digital subtraction angiography; CTA, computed tomography angiography; MRA, medical resonance angiography; CVS, central venous stenosis.

patients' medical records was not required due to the retrospective nature of the study. All data were anonymized to comply with the provisions of personal data protection legislation. No CVS-related symptoms (edema of the arms, head, face, and chest as well as dilated or varicose veins in the chest wall) were found during routine monitoring, and the pulsation at the arteriovenous anastomosis was normal. However, the patients had experienced the sensation of swelling in the ipsilateral upper limb during dialysis. The venous pressure either exceeded $150 \mathrm{mmHg}$ or had increased by $50 \mathrm{mmHg}$ upon the completion of dialysis; or both $(\mathrm{n}=52)$. After the appearance of abnormality during dialysis, a complete CDU was performed on the same day or the next day, which showed that the temporal waveform of the axillary venous flow spectrum (cardiac pulsation and respiratory phase) was "normal" which did not change to a flat waveform and the spectrum was clearly displayed (including a complete respiratory phase), and the acoustic data was complete. Subsequently, the presence of CVS $(\geq 50 \%$ stenosis) was confirmed by DSA the day after CDU. The exclusion criteria were as follows: (I) stent placement; (II) presence of multiple stenosis; and (III) placement of central venous catheter (CVC). A total of 37 patients participated in the final analysis (Figure 1), among whom 18 had CVS. Due to the small sample size, we did not group the participants according to the degree of stenosis; instead, all the CVS patients with a stenosis degree of $\geq 50 \%$ were categorized as the CVS group (including 11 males and 7 females, aged $37-74$ years, with a mean age of 57.72 years). In addition, 19 patients without CVS, as confirmed by DSA during the same period, were categorized as the control group (including 12 men and 7 women, aged 26-72 years, with an average age of 58.63 years).

\section{Instruments and methods}

\section{CDU scanner}

A Hi Vision Preirus ultrasonography system (Hitachi, Tokyo, Japan) with L74M 13-5 MHz and C715 5-1MHz probes and an Aplio 500 ultrasound system (Toshiba, Tokyo, Japan) with PLT-1005BT 14L5 $10 \mathrm{MHz}$ and PVT-375BT $6 \mathrm{C} 13.5 \mathrm{MHz}$ probes were used. 


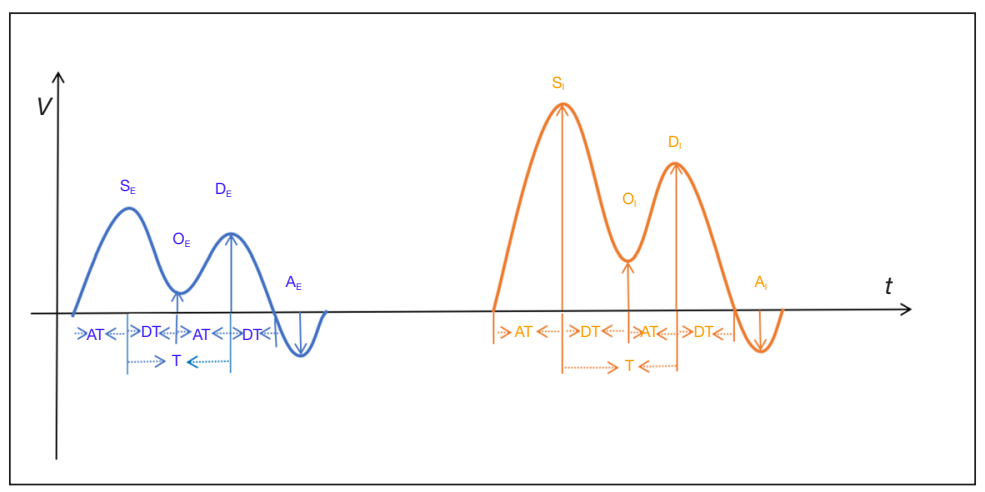

Figure 2 Schematic diagram of spectral waveforms of axillary vein. V corresponds to blood velocity, t to time; Blue corresponds to expiatory phase, orange to inspiratory phase; E corresponds to Expiration, I to inspiration; S corresponds to S-wave peak flow velocity, D to D-wave peak flow velocity, $\mathrm{O}$ to $\mathrm{O}$-wave flow velocity, A to A-wave trough flow velocity; AT corresponds to acceleration time, DT to acceleration time, $\mathrm{T}$ to time interval.

\section{Scanning}

The superior vena cava, innominate vein, subclavian vein, and distal segment of axillary vein were scanned via the supraclavicular and subclavian approaches and the supraclavicular fossa. During the examination, the patient was placed in a supine position after resting, and their head was tilted back or turned to the opposite side to that being examined; some patients felt discomfort during the examination but were still able tolerate and cooperate with the examination.

The examination conditions were adjusted when necessary. (I) The focus was placed at the level of the posterior wall of the vessel when B-mode clearly showed a non-echoic lumen. (II) The color sampling frame was set at an appropriate angle and size to obtain a good frame rate; the color gain and scale were adjusted to show the blood flow signals only within the lumen, without overflow. (III) The spectral Doppler angle $(\theta)$ was $<60$ degree and parallel to the direction of blood flow. The sampling site was placed in the middle of the lumen, with a volume of $1 / 3-1 / 2$ of the lumen when the vein was normal; when the veins were stenosed, the sample site was placed at the brightest part of the colored blood flow or the disordered site, with minimized sampling size.

When a stenotic site was found, the measurements included: (I) the diameters of the stenotic site and its adjacent segments; and (II) the peak flow velocity at the stenosis and in the region upstream to the stenosis.

\section{Axillary vein spectroscopy}

During resting respiration, the amplitude (i.e., flow velocity) of S-, D-, O-, and A-waves, the acceleration time and deceleration time of $\mathrm{S}$ - and D-waves, and the time interval between the peak of S-wave to the peak of $\mathrm{D}$-wave were measured during expiration (i.e., when the flow velocity is minimal) and during inspiration (i.e., when the flow velocity is maximal), respectively, within one respiratory phase (Figure 2). Since only temporal (heartbeat and respiratory phase) changes reflected by spectral morphology are focused on in routine clinical examinations and no parameter is measured, all the above parameters were acquired in this study for retrospective analysis using ImageJ (https:// imagej.nih.gov/ij) for late measurements of the spectrum, and a scale tool was used to calibrate each image before measurement. The measurements were performed by 2 ultrasonographers with more than 10 years of experience in vascular ultrasound. Each parameter was measured 3 times and averaged, and the two physicians were blind to the clinical diagnoses at the time of measurement.

All these parameters were divided into three categories: cardiac pulsation-driven parameters, respiration-driven parameters, and parameters affected by both cardiac pulsation and respiration.

Cardiac pulsation-driven parameters (measured during expiration, i.e., the minimum value during a single breath) included: $\mathrm{S}_{\mathrm{E}}$, S-wave peak flow velocity; $\mathrm{D}_{\mathrm{E}}$, D-wave peak flow velocity; $\mathrm{O}_{\mathrm{E}}, \mathrm{O}$-wave flow velocity; $\mathrm{A}_{\mathrm{E}}$, A-wave trough flow velocity; $\mathrm{S}_{\mathrm{E}} \mathrm{D}_{\mathrm{E}}$, S-wave peak flow velocity-D-wave peak flow velocity; $\mathrm{S}_{\mathrm{E}}$, $\mathrm{S}$-wave peak flow velocity-O-wave flow velocity; $\mathrm{D}_{\mathrm{E}}$ ', D-wave peak flow velocity-O-wave flow velocity; $\mathrm{S}_{\mathrm{EAT}}$, $\mathrm{S}$-wave acceleration time; $\mathrm{S}_{\mathrm{EDT}}$, $\mathrm{S}$-wave deceleration time; $\mathrm{D}_{\mathrm{EAT}}$, $\mathrm{D}$-wave acceleration time; $\mathrm{D}_{\mathrm{EDT}}$, $\mathrm{D}$-wave deceleration time; and $\mathrm{S}_{\mathrm{E}} \mathrm{D}_{\mathrm{E}} \mathrm{T}$, time interval from S-wave peak to D-wave peak. 
The respiration-driven parameters included the following: $\mathrm{S}_{\mathrm{I}} \mathrm{S}_{\mathrm{E}}, \mathrm{S}$-wave peak flow velocity during inspiration-S-wave peak flow velocity during expiration; $\mathrm{D}_{\mathrm{I}} \mathrm{D}_{\mathrm{E}}, \mathrm{D}$-wave peak flow velocity during inspiration-D-wave peak flow velocity during expiration; $\mathrm{O}_{\mathrm{I}} \mathrm{O}_{\mathrm{E}}, \mathrm{O}$-wave flow velocity at the end of inspiration-O-wave flow velocity at the end of expiration; $\mathrm{S}_{\mathrm{I}}^{\prime} \mathrm{S}_{\mathrm{E}}^{\prime}, \mathrm{S}_{\mathrm{I}}{ }^{-}-\mathrm{S}_{\mathrm{E}}^{\prime}$; and $\mathrm{D}_{\mathrm{I}}{ }^{\prime} \mathrm{D}_{\mathrm{E}}^{\prime}, \mathrm{D}_{\mathrm{I}}^{\prime}-\mathrm{D}_{\mathrm{E}}$ '.

Parameters affected by both cardiac pulsation and respiration (measured during inspiration, i.e., the maximum value during a single breath) included: $S_{\mathrm{I}}$, $\mathrm{S}$-wave peak flow velocity; $\mathrm{D}_{\mathrm{I}}$, D-wave peak flow velocity; $\mathrm{O}_{\mathrm{I}}$, O-wave flow velocity, $A_{I}$, A-wave trough flow velocity; $\mathrm{S}_{\mathrm{I}} \mathrm{D}_{\mathrm{I}}$, S-wave peak flow velocity-D-wave peak flow velocity; SI', S-wave peak flow velocity-O-wave flow velocity; DI', D-wave peak flow velocity-O-wave flow velocity; $\mathrm{S}_{\text {IAT }}$, $\mathrm{S}$-wave acceleration time; $\mathrm{S}_{\mathrm{IDT}}, \mathrm{S}$-wave deceleration time; $\mathrm{D}_{\mathrm{IAT}}, \mathrm{D}$-wave acceleration time; $\mathrm{D}_{\mathrm{IDT}}$, $\mathrm{D}$-wave deceleration time; and $\mathrm{S}_{\mathrm{I}} \mathrm{D}_{\mathrm{I}} \mathrm{T}$, time interval from $\mathrm{S}$-wave peak to $\mathrm{D}$-wave peak.

\section{Venography}

The examination was performed by a physician with 5 years of experience in vascular surgery, using an Artis Q ceiling system (Siemens, Munich, Germany), after they had fully reviewed the patient's clinical data and the participant had provided their informed consent. A vascular sheath was inserted through the cephalic vein (Seldinger's method), and a non-ionic contrast medium (Iohexol) was injected into the sheath, while digital subtraction was performed. The images were acquired at a frame rate of 1-3 images per second with posterior-anterior projection. The angiography revealed the blood return in the central veins (subclavian vein - innominate vein - superior vena cava) and identified the site, degree, and length of stenosis.

\section{CVS evaluation criteria}

The DSA technique is the gold standard method for CVS evaluation. The degree of stenosis was divided into 5 levels according to percent diameter stenosis (\%DS): normal, mild stenosis $(<50 \%)$, moderate stenosis $(50-69 \%)$, severe stenosis (70-99\%), and occlusion. The \%DS was calculated using the following formula: \% DS = (1 - stenosis diameter $/$ reference diameter) $\times 100 \%$, where the stenosis diameter is the diameter of the vessel at the narrowest point of the lumen and the reference diameter is the original diameter of the vessel at the stenosis site, which was estimated by the system according to the diameters of the normal vessels at both ends of the stenotic segment.
The CVS was evaluated by ultrasound (14), according to an internal diameter of $<2.0 \mathrm{~mm}$, a $50 \%$ reduction in the internal diameter of the stenotic site compared with the adjacent segments, and a flow velocity ratio of $>2.5$.

\section{Statistical analysis}

Statistical analysis was performed using the software SPSS 25.0 (IBM Corp., Armonk, NY, USA). Normally distributed measurement data were presented as $(\mathrm{X} \pm \mathrm{SD})$.

\section{Construction of a comprehensive CVSI}

Independent sample $t$-test was used to screen each parameter of the axillary venous blood flow spectrum. Principal component analysis (PCA) was performed on the parameters with $\mathrm{P}<0.05$ in independent sample $t$-test, and principal components with eigenvalues $\geq 1$ were selected. After nonstandardized conversion of original parameters of principal components, an index model of ultrasonic evaluation indexes was constructed, which was defined as CVSI.

\section{Analysis of CVSI diagnostic value}

The receiver operating characteristic (ROC) curve was applied to analyze the diagnostic value of CVSI.

\section{Results}

Among the 37 suspected CVS cases, CVS was confirmed by DSA in 18 cases, which was directly detected by CDU in 5 cases, while the remaining 13 CVS cases were not detected by CDU because the stenosis site could not be clearly displayed and the wave number spectrum (cardiac pulsation and respiratory phase) in the upstream region did not disappear, yielding a detection rate of $27.78 \%$. Central veins with stenotic sites included the superior vena cava, innominate vein, and subclavian vein (Table 1).

\section{Construction of CVSI}

\section{Screening of CVSI-related parameters}

Independent sample $t$-test was used to screen the relevant parameters, and 9 parameters including $\mathrm{O}_{\mathrm{E}}, \mathrm{O}_{\mathrm{I}}, \mathrm{S}_{\mathrm{I}}-\mathrm{D}_{\mathrm{I}}$, $\mathrm{S}_{\mathrm{I}} \mathrm{S}_{\mathrm{E}}, \mathrm{S}_{\mathrm{EAT}}, \mathrm{D}_{\mathrm{EDT}}, \mathrm{S}_{\mathrm{IDT}}, \mathrm{S}_{\mathrm{IAT}}$, and $\mathrm{S}_{\mathrm{I}} \mathrm{D}_{\mathrm{I}} \mathrm{T}$ showed statistically significant differences (all $\mathrm{P}<0.05)$ (Table 2).

\section{Construction of a ultrasonic comprehensive evaluation model of central venous stenosis using PCA}

These 9 parameters were subjected to PCA to establish 
Table 1 Distribution of stenosis sites in central veins and their detection on ultrasound

\begin{tabular}{lccc}
\hline Site & CVS case $(\mathrm{n})$ & CVS case detected by ultrasound $(\mathrm{n})$ & Detection rate $(\%)$ \\
\hline Superior vena cava & 1 & 0 & 0 \\
Innominate vein & 9 & 2 & 22.22 \\
Subclavian vein & 8 & 3 & 37.50 \\
Total & 18 & 5 & 27.78 \\
\hline
\end{tabular}

CVS, central venous stenosis.

a Ultrasonic comprehensive evaluation index, which was defined as central venous stenosis index (CVSI), and the results are shown in Tables 3,4.

The contribution rates of the first 4 eigenvectors reached $80.510 \%$. It was assumed that the first 4 eigenvectors were able to represent most of the information of the original index, so it was appropriate to extract the first 4 eigenvectors to construct the prognostic CVSI model. Thus, a comprehensive evaluation model $\left(\mathrm{CVSI}=\mathrm{Z}_{1}+\mathrm{Z}_{2}+\mathrm{Z}_{3}+\mathrm{Z}_{4}\right)$ was obtained after the non-standardized transformation of the original index: CVSI $=0.0275 \mathrm{X}_{1}+0.0355 \mathrm{X}_{2}-0.0194 \mathrm{X}_{3}$ $+0.0119 \mathrm{X}_{4}+0.0084 \mathrm{X}_{5}+0.0065 \mathrm{X}_{6}+0.0049 \mathrm{X}_{7}+0.0007 \mathrm{X}_{8}+$ $0.0027 \mathrm{X}_{9}$.

The final CVSI range of our participants was from 3.73 to 10.38 , with a mean of $6.44 \pm 2.08$.

\section{Analysis of CVSI diagnostic value}

The ROC curve was applied to analyze the diagnostic value of CVSI (Figure 3). For the CVS group and control group, the area under the ROC curve (AUC) of CVSI was 0.965 [95\% confidence interval $(\mathrm{CI}): 0.915$ to 0.998$](\mathrm{P}<0.001)$, suggesting CVSI has a diagnostic value for resting-state asymptomatic CVS in stage 5 CKD patients undergoing hemodialysis via RCAVF (Table 5).

Based on the maximum value of the Youden index (YI) $(\mathrm{YI}=$ sensitivity + specificity -1$)$, when the CVSI cutoff value was 7.13 , the maximum YI was 0.842 , and its diagnostic sensitivity and specificity were $100 \%$ and $84.2 \%$, respectively.

\section{Discussion}

Stenosis is a common cause of vascular access dysfunction during hemodialysis. Most of these stenoses are near the anastomosis in the peripheral segment of the access, where the venous pressure can be dramatically higher than that in the central veins (15). Therefore, a possible co-existing stenosis in the central veins can be easily neglected when a cause of restricted blood flow due to peripheral venous stenosis is identified. Idiopathic stenosis can also exist in the central veins. In the physiological state, there might be no clinical symptoms such as swelling of the arm, face, or chest wall, even if severe stenosis occurs in a central vein; however, after a vascular access for hemodialysis is created, the increase in blood flow will exceed the normal physiological load, causing an increase in venous pressure. In addition, high blood volume and disturbed blood flow after a vascular access is established can promote intimal hyperplasia, which aggravates stenosis and thereby limits the blood flow through the vascular access.

Although ultrasound is the preferred modality for the monitoring and maintenance of vascular access after its creation, there are technical difficulties in the examination of central veins. First, due to the narrow space and bony structures in the supraclavicular and suprasternal regions, the subclavian vein and innominate vein cannot be easily revealed. In our series, a convex array probe, in addition to the conventional linear array high-frequency probe, was applied when scanning the above regions. The detection rate of CVS in our current study was only $27.78 \%$, which was quite similar to that reported by Agarwal et al. (16). Therefore, the diagnosis of CVS by observing the changes in venous diameter at the stenosis site and by calculating the ratio of blood flow velocity at the stenosis site to that in its upstream region is featured by low detection rate and high rate of missed diagnosis. In addition, the method of inferring stenosis by indirect ultrasound signs (17) (i.e., the disappearance of cardiac pulsation-driven and respirationdriven time phases in the upstream region) can only detect severe stenosis. However, such a qualitative method has high specificity but low sensitivity and may miss some of the CVS in which the cardiac pulsation-driven and respirationdriven time phases change but do not disappear (Figures 4,5).

Therefore, our current study was designed to improve the detection rate of resting asymptomatic CVS by quantifying 
Table 2 Screening of CVSI-related parameters

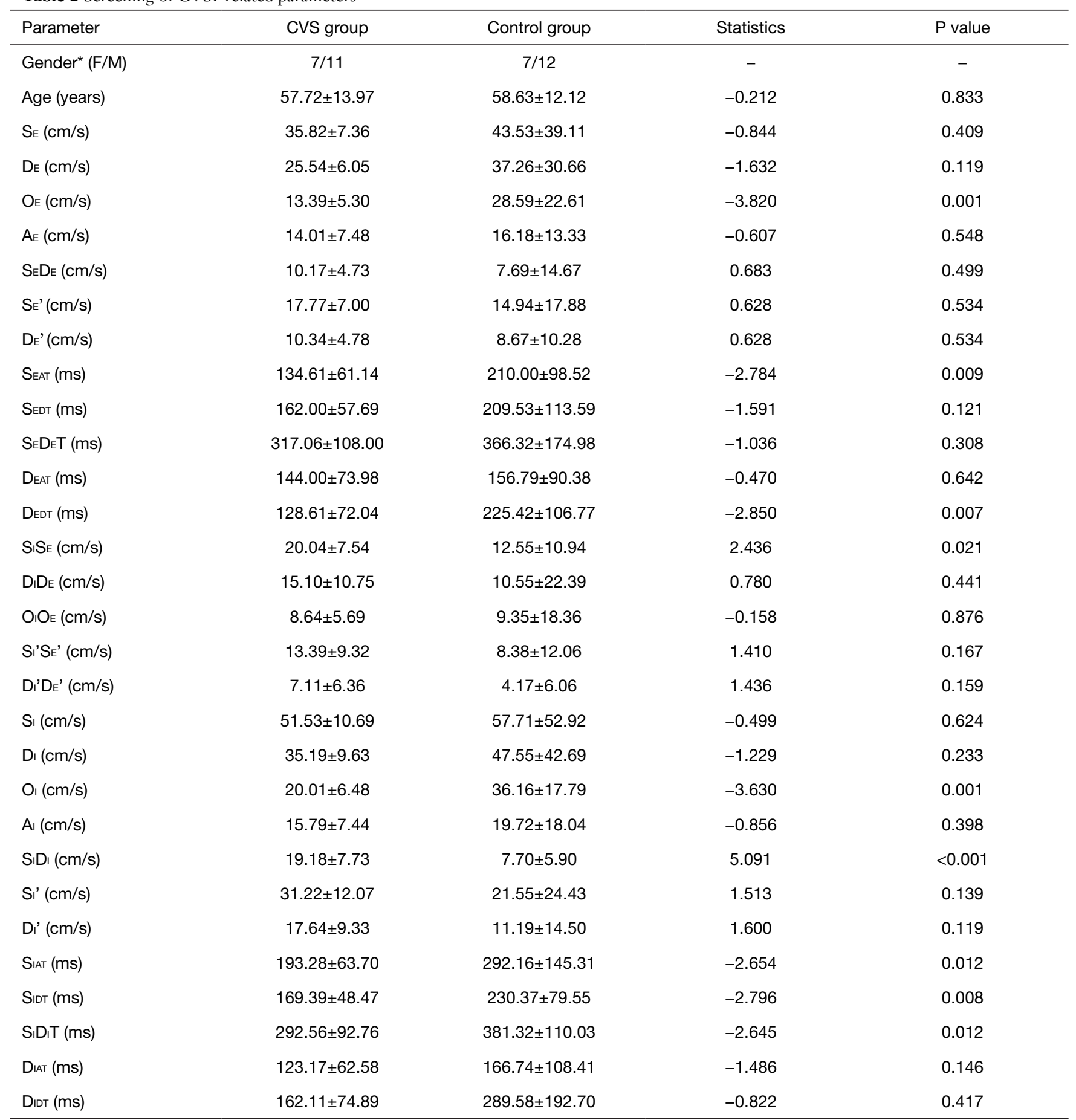

*, Chi-square statistics; the remaining statistics are t or t' values. CVSI, central venous stenosis index.

multiple parameters associated with cardiac pulsation-driven and respiration-driven time phases on the axillary venous flow spectrum in the upstream region of CVS.

At the beginning of this study, the parameters were divided into three categories, namely those affected by cardiac pulsation, those affected by respiration, and those affected by both cardiac pulsation and respiration, in the hope of discovering which aspects of CVS interfere with 
Table 3 Eigenvectors, contribution rates, and cumulative contribution rates

\begin{tabular}{lccc}
\hline Principal component & Value of eigenvectors & Contribution rates (\%) & Cumulative contribution rates \\
\hline 1 & 3.078 & 34.199 & 34.199 \\
2 & 1.920 & 21.331 & 55.530 \\
3 & 1.177 & 13.082 & 68.612 \\
4 & 1.071 & 11.899 & 80.510 \\
\hline
\end{tabular}

Table 4 Eigenvalues for the correlation matrix in PCA

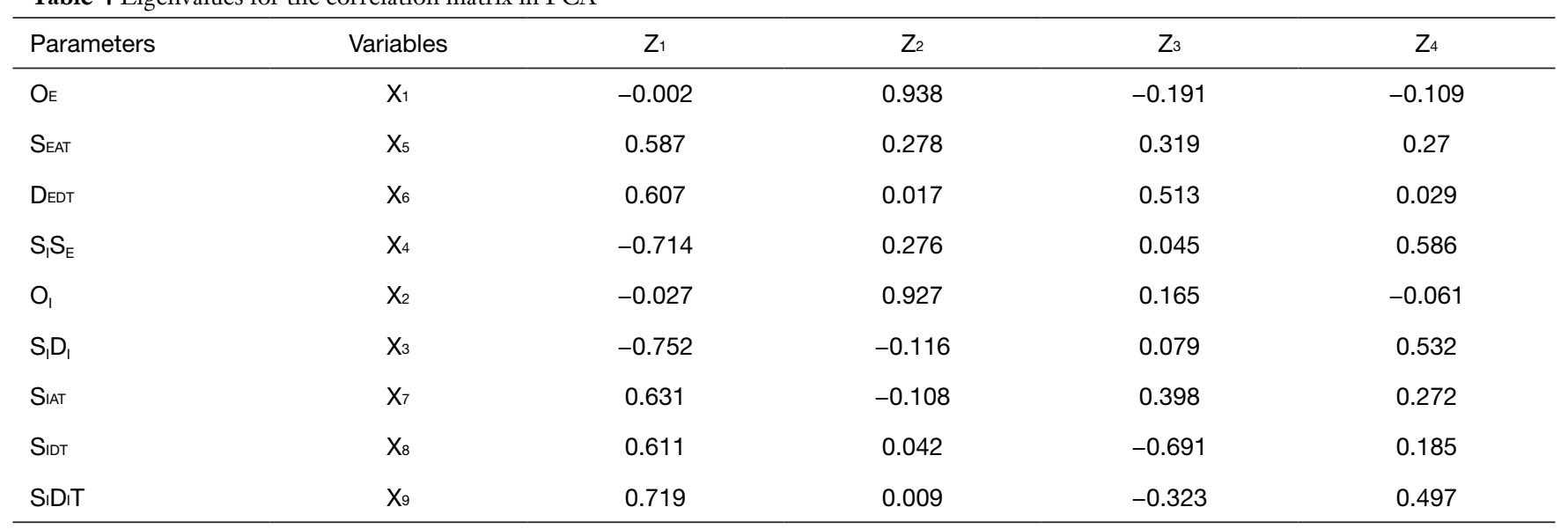

PCA, principal component analysis.

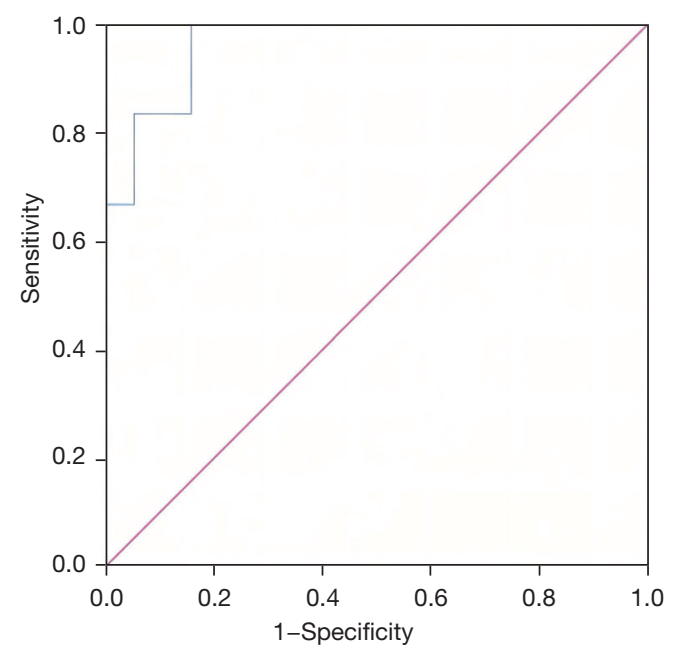

Figure 3 Analysis of the diagnostic value of CVSI using ROC curves. CVSI, central venous stenosis index; ROC, receiver operating characteristic.

blood return and identifying simple and effective thresholds of the quantified parameters. However, our statistical analysis suggested that CVS did not affect only one of these three categories of parameters; rather, it affected all of them, and the affected parameters could be divided into two categories: velocity and time.

The normal axillary venous flow spectral waveform is similar to the central venous flow spectral waveform in the thoracic cavity and shows changes in two temporal phases, namely, the temporal phases of cardiac pulsation and respiration. The temporal changes in the spectral waveform include the following waveforms (18): two distinct antegrade waves ( $\mathrm{S}$ and $\mathrm{D}$ waves, which arise during ventricular systole and early diastole, respectively) and two small retrograde waves or reduced antegrade waves (A and $\mathrm{O}$ waves, which arise after atrial systole and second heart sound, respectively).

In our current study, PCA showed that the largest contribution rate $(34.199 \%)$ to CVS was from the time parameters, which included parameters influenced by cardiac pulsation $\left(\mathrm{S}_{\mathrm{EAT}}, \mathrm{D}_{\mathrm{EDT}}\right)$ and by respiration $\left(\mathrm{S}_{\mathrm{IAT}}, \mathrm{S}_{\mathrm{IDT}}\right.$, and $\left.\mathrm{S}_{\mathrm{I}} \mathrm{D}_{\mathrm{I}} \mathrm{T}\right)$; in other words, the factor with the greatest effect on upstream blood return was time. Pham et al. (17) suggested that the upstream subclavian and axillary venous blood flow spectra in CVS patients lacked temporal variations, 
Table 5 Analysis of the predictive power of CVSI

\begin{tabular}{lcccc}
\hline Variable & AUC & Standard error & P value & 95\% Cl of AUC \\
\hline CVSI & 0.965 & 0.026 & $<0.001$ & $0.915-0.998$ \\
\hline
\end{tabular}

CVSI, central venous stenosis index; AUC, area under the curve; $\mathrm{Cl}$, confidence interval.

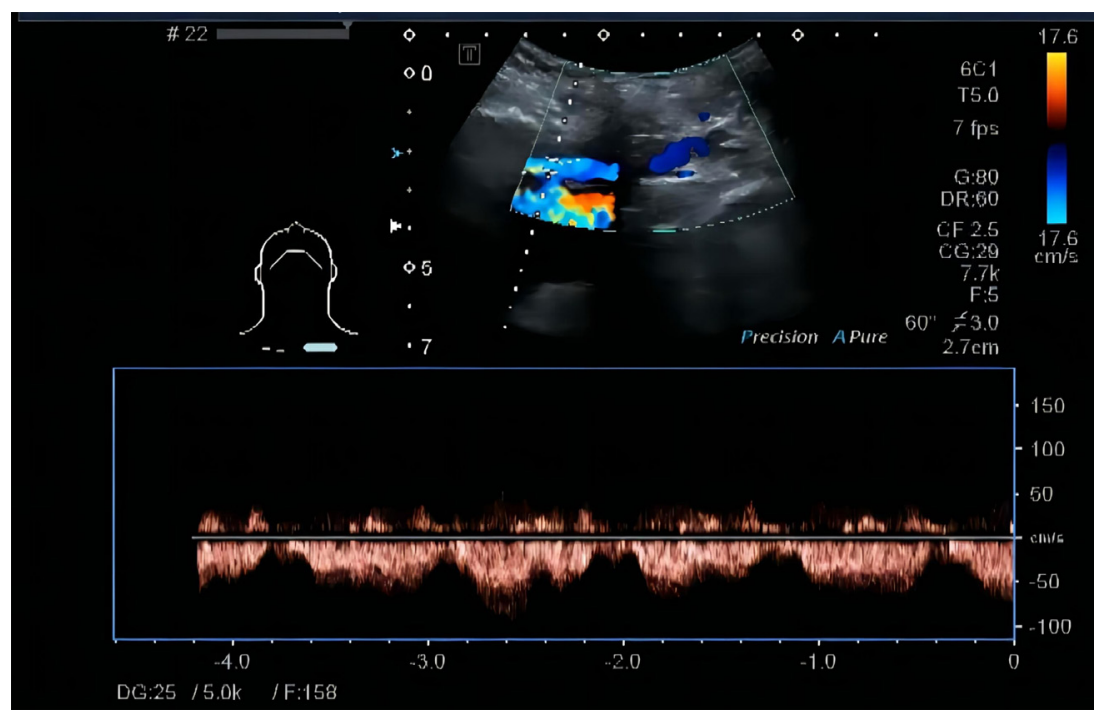

Figure 4 Spectral waveforms of left axillary vein when stenosis occurs in left innominate vein in a patient on maintenance hemodialysis via an RCAVF on left forearm. RCAVF, radiocephalic arteriovenous fistula.

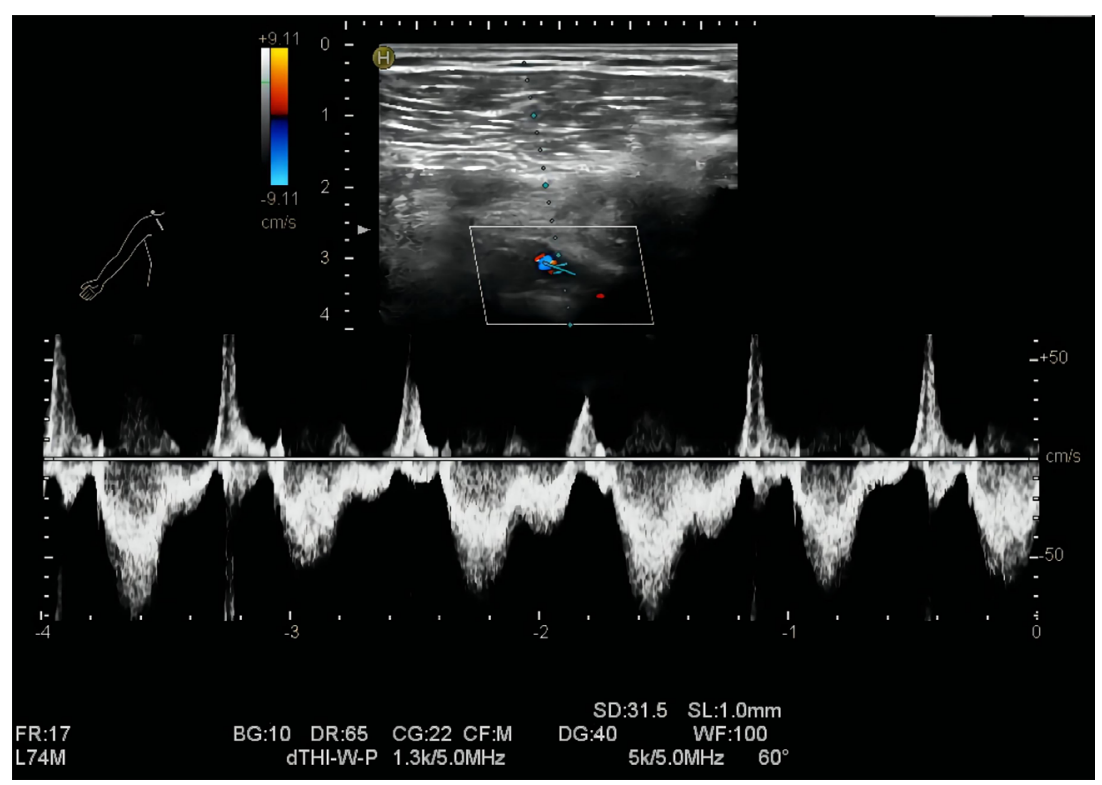

Figure 5 Spectral waveforms of right axillary vein in a non-CVS patient on maintenance hemodialysis via an RCAVF on right forearm. CVS, central venous stenosis; RCAVF, radiocephalic arteriovenous fistula. 
showing a stutter-like change in waveform including reduced amplitude and diminished volatility. Such a waveform change results from the extreme changes in intraventricular pressure difference and vasodilatability, forming wavewave fusion. In our series, however, the waveforms formed before the limb signs occurred, which were quite different from the transition of normal waveforms. Most of the velocity parameters in the CVS group were not significantly different from those in normal controls (Table 2), while $\mathrm{Z}_{1}$ time parameters $\left(S_{\text {EAT }}, D_{\text {EDT }}, S_{\text {IAT }}, S_{\text {IDT }}, S_{I} D_{I} T\right), Z_{2}$ velocity parameters $\left(\mathrm{O}_{\mathrm{E}}, \mathrm{O}_{\mathrm{I}}\right)$, and $Z_{3}$ time parameters $\left(\mathrm{D}_{\mathrm{EDT}}\right)$ were significantly different from controls and contributed to the detection of CVS by $34.199 \%, 21.331 \%$, and $13.082 \%$, respectively (Tables 2-4).

Normally, the right atrial pressure is about $0 \mathrm{mmHg}$; there is no resistance in the veins when the central veins in the thoracic cavity are filled; and the venous pressure in the axillary veins of the upper limbs is about $+6 \mathrm{mmHg}$. Therefore, blood in the central veins can flow back into the right atrium smoothly (19). However, when there is a stenosis site in the central veins, the pressure difference at the stenosis site will increases, according to Poiseuille's law $(\mathrm{F}=\pi \Delta \operatorname{Pr} 4 / 8 \eta \mathrm{L})$. Since the right atrial pressure is $0 \mathrm{mmHg}$, the pressure at the upper end of the stenosis site increases. As a result, the pressure difference between the upstream region of the stenosis site and the upper end of the stenosis site decreases, resulting in shortened acceleration time and deceleration time. Meanwhile, the narrowing of the vascular lumen and the increase in friction at the stenosis site lead to a decrease in vascular conductivity and an increase in resistance. The $\mathrm{O}$-wave appears at the beginning of the isovolumic diastolic phase after the second heart sound appear and is located at the aortic dicrotic notch, where the axillary venous pressure is low and the venous dilatability is high (20,21). Accordingly, blood accumulation and vasodilatation occur at this location, leading to reduced flow velocity during the $\mathrm{O}$-wave in the upstream region.

The $Z_{4}$ velocity parameters $\left(S_{I} S_{E}\right.$ and $\left.S_{I} D_{I}\right)$ were significantly different from controls and contributed $11.899 \%$ of the detection of CVS, respectively (Tables 2-4). During the body circulation, the large diameter of the veins and the specific structures of venous valves facilitate venous return. However, the venous pressure is too low to promote adequate blood return. Therefore, the "pump" structures, including the muscle "pump", respiratory "pump", and sympathetic modulation of venous contraction, outside the body circulation assist and promote venous blood return (20). For the central veins (including subclavian vein, innominate vein, superior vena cava), through which blood via the RCAVF returns, the blood return is usually facilitated by the respiratory "pump". During inspiration, the intrathoracic pressure decreases and the "pump" acts to cause the intrathoracic veins (subclavian vein, innominate vein, superior vena cava) to dilate, leading to higher $S_{I}$ than $S_{E}$ and promoting the flow of more blood into the right atrium. Notably, since the venous dilatation is more pronounced than the change in pressure difference, the change in velocity is not significant. However, CVS may be caused by venous compression, excessive intimal hyperplasia, and/or thrombosis. All of these factors can lead to change in pressure difference in the central vein when the intrathoracic pressure decreases, but the lumen diameter cannot expand accordingly. As a result, the preand post-expiratory velocities (i.e., $\mathrm{S}_{\mathrm{I}}$ and $\mathrm{S}_{\mathrm{E}}$ ) change more than the diameter change to maintain blood flow; in other words, $\mathrm{S}_{\mathrm{I}} \mathrm{S}_{\mathrm{E}}$ increases during CVS. The D-wave in the spectrum appears in the early phase of ventricular diastole, during which the right atrial pressure is high and the arterial pressure driving the blood circulation begins to fall, resulting in a decrease in the difference between peripheral venous pressure and right atrial pressure (20). As a result, the change in the $\mathrm{D}$-wave during inspiration is not significant, which in turn results in a greater $S_{I}$ than $D_{I}$ during inspiration in CVS patients, that is, an increase in the $S_{I} D_{I}$ value, which is statistically significant from that in the control group.

Therefore, it was appropriate to construct a CVSI model by using the four eigenvectors including $Z_{1}, Z_{2}, Z_{3}$, and $Z_{4}$. The ROC curve was used to analyze the diagnostic value of CVSI. The AUC of the CVSI was 0.965, suggesting that CVSI has a certain diagnostic value for hemodialysisrelated CVS in CKD patients. When the CVSI cutoff value was 7.13 , the maximum YI was 0.842 , and its diagnostic sensitivity and specificity were $100 \%$ and $84.2 \%$, respectively. Compared with the direct signs at the CVS site, CVSI is more sensitive while maintaining a high specificity.

Although the CVSI, which is established based on the upstream flow spectrum of CVS, markedly improves the detection rate of asymptomatic CVS and avoids the underdiagnosis and delayed prevention and treatment of CVS during hemodialysis vascular access monitoring, it has a limited role in determining the site, extent, and etiology of stenosis and in informing further endovascular treatment, for which angiography is still indispensable. Although the severity of CVS was not graded in our current study, the 
spectral waveforms were quite similar, suggesting that the hemodynamic changes were similar. Therefore, the impact of the severity of stenosis on data analysis might have been neglected. However, due to the small sample size in our current study, the established CVSI model could not be externally validated. In summary, the model is promising for clinical application and deserves further validation through larger sample research.

\section{Acknowledgments}

Funding: This work was supported by Leading Project Foundation of Science and Technology, Fujian Province (No. 2020Y0024).

\section{Footnote}

Reporting Checklist: The authors have completed the STARD reporting checklist. Available at https://atm.amegroups. com/article/view/10.21037/atm-22-160/rc

Data Sharing Statement: Available at https://atm.amegroups. com/article/view/10.21037/atm-22-160/dss

Conflicts of Interest: All authors have completed the ICMJE uniform disclosure form (available at https://atm. amegroups.com/article/view/10.21037/atm-22-160/coif). All authors report this work was supported by Leading Project Foundation of Science and Technology, Fujian Province (No. 2020Y0024). The authors have no other conflicts of interest to declare.

Ethical Statement: The authors are accountable for all aspects of the work in ensuring that questions related to the accuracy or integrity of any part of the work are appropriately investigated and resolved. This study was approved by the Ethics Committee of the First Affiliated Hospital of Fujian Medical University [MRCTA, ECFAH of FMU (2020)189]. Informed consent to review patients' medical records was not required due to the retrospective nature of the study. All data were anonymized to comply with the provisions of personal data protection legislation. This study adhered to the tenets of the Declaration of Helsinki (as revised in 2013).

Open Access Statement: This is an Open Access article distributed in accordance with the Creative Commons Attribution-NonCommercial-NoDerivs 4.0 International
License (CC BY-NC-ND 4.0), which permits the noncommercial replication and distribution of the article with the strict proviso that no changes or edits are made and the original work is properly cited (including links to both the formal publication through the relevant DOI and the license). See: https://creativecommons.org/licenses/by-nc-nd/4.0/.

\section{References}

1. Vascular Access Work Group. Clinical practice guidelines for vascular access. Am J Kidney Dis 2006;48 Suppl $1:$ S248-73.

2. Schmidli J, Widmer MK, Basile C, et al. Editor's Choice Vascular Access: 2018 Clinical Practice Guidelines of the European Society for Vascular Surgery (ESVS). Eur J Vasc Endovasc Surg 2018;55:757-818.

3. Tedla FM, Clerger G, Distant D, et al. Prevalence of Central Vein Stenosis in Patients Referred for Vein Mapping. Clin J Am Soc Nephrol 2018;13:1063-8.

4. Ehrie JM, Sammarco TE, Chittams JL, et al. Unmasking of Previously Asymptomatic Central Venous Stenosis following Percutaneous Transluminal Angioplasty of Hemodialysis Access. J Vasc Interv Radiol 2017;28:1409-14.

5. Trerotola SO, Kothari S, Sammarco TE, et al. Central venous stenosis is more often symptomatic in hemodialysis patients with grafts compared with fistulas. J Vasc Interv Radiol 2015;26:240-6.

6. Levit RD, Cohen RM, Kwak A, et al. Asymptomatic central venous stenosis in hemodialysis patients. Radiology 2006;238:1051-6.

7. Arslanian J. Monitoring and surveillance of the hemodialysis vascular access. Nephrol News Issues 2015;29:38-42.

8. Dumars MC, Thompson WE, Bluth EI, et al. Management of suspected hemodialysis graft dysfunction: usefulness of diagnostic US. Radiology 2002;222:103-7.

9. Labropoulos N, Borge M, Pierce K, et al. Criteria for defining significant central vein stenosis with duplex ultrasound. J Vasc Surg 2007;46:101-7.

10. Doelman C, Duijm LE, Liem YS, et al. Stenosis detection in failing hemodialysis access fistulas and grafts: comparison of color Doppler ultrasonography, contrastenhanced magnetic resonance angiography, and digital subtraction angiography. J Vasc Surg 2005;42:739-46.

11. Sivanesan S, How TV, Black RA, et al. Flow patterns in the radiocephalic arteriovenous fistula: an in vitro study. J Biomech 1999;32:915-25.

12. Modabber M, Kundu S. Central venous disease in 


\section{Page 12 of 12}

hemodialysis patients: an update. Cardiovasc Intervent Radiol 2013;36:898-903.

13. Agarwal AK, Haddad NJ, Khabiri H. How should symptomatic central vein stenosis be managed in hemodialysis patients? Semin Dial 2014;27:278-81.

14. Lu T, Younes HK, Bechara CF, et al. Detection of central venous stenosis in hemodialysis patients: a comparative study between intravascular ultrasound and venography. Journal of Vascular Surgery 2015;61:52S.

15. Sullivan KL, Besarab A, Bonn J, et al. Hemodynamics of failing dialysis grafts. Radiology 1993;186:867-72.

16. Agarwal AK. Central vein stenosis. Am J Kidney Dis 2013;61:1001-15.

17. Pham XD, Ihenachor EJ, Wu H, et al. Significance of Blunted Venous Waveforms Seen on Upper Extremity

Cite this article as: $\mathrm{Xu} \mathrm{X,} \mathrm{Zhuang} \mathrm{Y,} \mathrm{Zeng} \mathrm{J,} \mathrm{Cai} \mathrm{F,} \mathrm{He} \mathrm{T,}$ Wu J, Chen C, Zou Z, Zhang X, Lv G. Value of a quantitative model of axillary venous blood flow spectrum for the detection of central venous stenosis in patients undergoing hemodialysis via radiocephalic arteriovenous fistula. Ann Transl Med 2022;10(2):77. doi: 10.21037/atm-22-160

\section{Xu et al. Quantitative model of axillary venous spectrum in CVS}

Ultrasound. Ann Vasc Surg 2017;42:32-8.

18. Maeda T, Matsuzaki M, Shiomi K, et al. Characteristics of blood flow velocity patterns of central systemic veins in healthy adults assessed by Doppler echocardiography. Jpn Circ J 1991;55:535-42.

19. Silverthorn DU. Human Physioloy: An Integrated Approach (8th Edition). San Francisco: Pearson, 2019.

20. Marieb EN, Hoehn K. Human Anatomy \& Physiology (9th Edition). San Francisco: Pearson, 2013.

21. Shier D, Butler J, Lewis R. Hole's Human Anatomy \& Physiology (15th Edition). New York: McGraw-Hill Education, 2019.

(English Language Editor: J. Jones) 